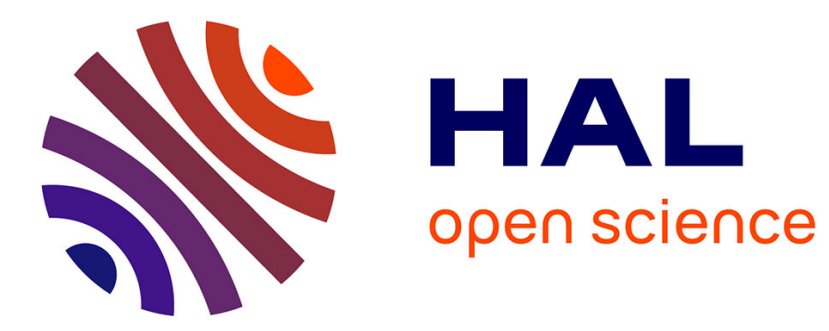

\title{
Coupled vibrations of a meniscus and liquid films
}

Jacopo Seiwert, Juliette Pierre, Benjamin Dollet

\section{To cite this version:}

Jacopo Seiwert, Juliette Pierre, Benjamin Dollet. Coupled vibrations of a meniscus and liquid films. Journal of Fluid Mechanics, 2016, 788, pp.183-208. 10.1017/jfm.2015.674 . hal-01247731

\section{HAL Id: hal-01247731 \\ https://hal.science/hal-01247731}

Submitted on 30 Sep 2016

HAL is a multi-disciplinary open access archive for the deposit and dissemination of scientific research documents, whether they are published or not. The documents may come from teaching and research institutions in France or abroad, or from public or private research centers.
L'archive ouverte pluridisciplinaire HAL, est destinée au dépôt et à la diffusion de documents scientifiques de niveau recherche, publiés ou non, émanant des établissements d'enseignement et de recherche français ou étrangers, des laboratoires publics ou privés. 


\title{
Coupled vibrations of a meniscus and liquid films
}

\author{
By JACOPO SEIWERT, JULIETTE PIERRE \\ AND BENJAMIN DOLLET $\dagger$ \\ Institut de Physique de Rennes, UMR 6251 CNRS/Université Rennes 1, Campus Beaulieu, \\ Bâtiment 11A, 35042 Rennes Cedex, France
}

(Received 30 September 2016)

We investigate the vibration properties of a circular horizontal film, that is bounded by a meniscus (or Plateau border) and suspended to two catenary films. The suspending films act as capillary springs, and the system is thus free to oscillate around its equilibrium position. We study successively its free and forced oscillations. In our experiments, we track simultaneously the positions of the Plateau border and of the film. The model that we present predicts the eigenfrequency of the system, and its resonance characteristics (in forced oscillations). In particular, we show that the dynamics of both the Plateau border and the film have to be taken into account, in order to provide an accurate prediction of the oscillation frequency.

\section{Introduction}

Liquid foams are dense dispersions of gas bubbles in a continuous liquid phase containing surfactants to ensure their stability. The bubbles are in contact through thin soap films, supported by an interconnected networks of liquid channels (also called Plateau borders). Foams exhibit a peculiar rheology, with a finite yield stress and shear modulus, a high effective viscosity and a non-Newtonian behavior. The link between those characteristics, which make them useful in a variety of industrial applications (Stevenson, 2012), and their structure has has been thoroughly investigated, both experimentally and theoretically, in the regime of small to intermediate strain rates and frequencies (up to a few tens of Hz) (see Cohen-Addad, Höhler \& Pitois, 2013, Dollet \& Raufaste, 2014 for recent reviews).

These shear rates and frequencies remain lower, however, than what is achieved in many industrial applications, such as the mitigation of shock and blast waves by aqueous foams (Goldfarb et al., 1997, Britan, Liverts \& Ben-Dor, 2009, Del Prete et al., 2013), or the design of new probes of liquid foams based on their acoustic response. A related subject is the propagation of acoustic waves through foams, which has been surprisingly considered only rather recently (Moxon, Torrance \& Richardson, 1988, Mujica \& Fauve, 2002, Kann, 2005, Pierre et al., 2013, Pierre, Dollet \& Leroy, 2014). Most experimental studies (Mujica \& Fauve, 2002, Pierre et al., 2013) report low values of the speed of sound (of the order of $50 \mathrm{~m} / \mathrm{s}$ ), in agreement with mean field models that predict the speed of sound from the average density and compressibility of the foam (Wood, 1944). However, much higher sound velocities (of the order of $200 \mathrm{~m} / \mathrm{s}$ ) have also been measured (Moxon, Torrance \& Richardson, 1988), and dedicated models (Kann, 2005) have been proposed, describing the sound propagation as occurring in the gas phase only, except for

$\dagger$ Email address for correspondence: benjamin.dollet@univ-rennes1.fr 
the additional inertia provided by the soap films. These different viewpoints have been unified in a recent study (Pierre, Dollet \& Leroy, 2014) which proposed a model based on a description of the vibration of the foam structure in response to the propagation of a pressure wave. It showed that at low frequency and/or small bubble size, the films and Plateau borders vibrate in phase and with the same amplitude, justifying the mean field approach. At large frequency and/or large bubble size, the vibration amplitude of the Plateau borders is negligible compared to that of the soap films, because of their huge difference of inertia, thereby justifying the model by Kann, 2005.

However, although the model by Pierre, Dollet \& Leroy, 2014 gives a satisfactory agreement with experimental measurements, it is not possible to check in situ whether the vibrational response of the films and Plateau borders agrees with the model, because of the opacity of the foams and the high frequencies. To do so, an experiment where the coupled vibrations of films and Plateau borders in response to an acoustic forcing can be directly visualised and measured is required.

Film vibration has been investigated by several groups: see for example Taylor, 1959, Couder, Chomaz \& Rabaud, 1989, Afenchenko et al., 1998, Vega, Higuera \& Weidman, 1998, Boudaoud, Couder \& Ben Amar, 1999, Elias, Hutzler \& Ferreira, 2007, Kosgodagan Acharige, Elias \& Derec, 2014. Taylor, 1959 introduced the possible modes of vibration for a film: a symmetric one, with thickness modulations, and an asymmetric bending mode, whereby the film retains a constant thickness. Films usually vibrate in a bending mode that is less dissipative than the symmetric mode. At low frequencies, simple flexural waves are observed; for a circular film, they take the form of circular rings. At higher frequencies, vortical structures develop inside the vibrating films (Couder, Chomaz \& Rabaud, 1989, Afenchenko et al., 1998, Vega, Higuera \& Weidman, 1998, Elias, Hutzler \& Ferreira, 2007). At high amplitudes, nonlinear effects take place, like self-adaptation of the film thickness to adopt a resonant configuration whatever the frequency (Boudaoud, Couder \& Ben Amar, 1999), which eventually leads to film rupture (Drenckhan et al., 2008).

All the aforementioned experiments were done with films tethered to a rigid frame. There are also some scattered observations about the coupled vibrations of films and Plateau borders. As a model system for foam rheology, Besson \& Debrégeas, 2007 studied the oscillations of two soap bubbles in contact, forced by a sinusoidal modulation of the distance between their two centers, and they quantified the modulation of the contact angle between the two bubbles. However, they did not study the vibration of the film separating the two bubbles. Hutzler et al., 2008 studied a Plateau border that is created following a topological rearrangement between adjacent films. They showed that the new Plateau border grows and displays free oscillations, with a characteristic frequency and damping, and suggested that air inertia is crucial to understand this dynamics, but no systematic study of the coupled vibration of a free film connected to a Plateau border has been undertaken.

Several works currently attempt to rationalise the coupled dynamics of the air, the soap films and the Plateau border. These studies differ by the geometry investigated and by the amplitude of the forcing. Elias et al., 2015 investigate the case of a linear Plateau border transversally vibrated, and measure and model the dispersion relation of the transverse waves for a low amplitude of forcing. For a higher forcing amplitude, Cohen, Fraysse \& Raufaste, 2015 observe the modulation of the cross-sectional area of the Plateau border in response to the vibration. In both studies the Plateau border is long enough so that several wavelengths take place along the Plateau border. In this paper, we address this issue on a model minimal configuration, the diaboloid (Salkin et al., 2014), consisting of two films (henceforth called the suspending films), each connected 
(a)

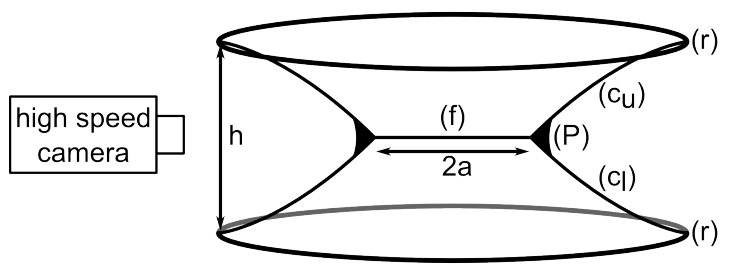

(b)

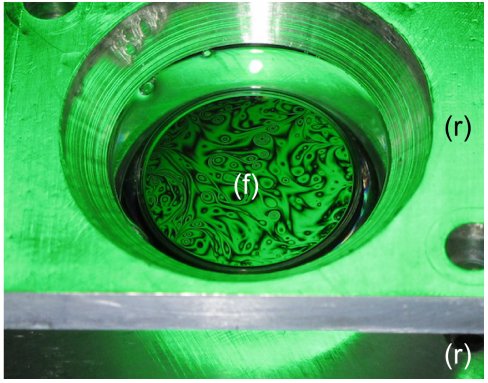

Figure 1. (a): Experimental setup (not to scale). The suspended film (f), of thickness $e \approx 0.5-1.5 \mu \mathrm{m}$ and diameter $2 a \approx 14-20 \mathrm{~mm}$, is suspended between two aluminum rings (r) (22 $\mathrm{mm}$ in diameter) placed a distance $h$ apart via an upper and a lower suspending film $\left(c_{u}\right)$ and $\left(c_{l}\right)$. The three films meet at the Plateau border $(\mathrm{P})$ at an angle of approximately $120^{\circ}$. $(\mathrm{P})$ is backlit and its profile is monitored by a high speed camera. (b): Close-up of the rings. On this image, the suspended film (f) is lit with a monochromatic lamp, which produces the interference pattern seen on the picture.

to a circular ring and taking the shape of a catenoid, and connected to each other by a planar soap film, henceforth called the suspended film (Figs. 1a and 2a). This is a model system for our purposes, since the liquid meniscus at the intersection of three films mimics the Plateau border in foams, and the suspended film is freely suspended to this meniscus (as opposed to rigidly tethered to a solid ring). This configuration has been used by Seiwert et al., 2013 to study the behaviour of such a suspended film under extension.

Our paper is organised as follows. In Sec. 2, we describe our setup and we quantify the eigenfrequency and damping of the free oscillations of the Plateau border. In Sec. 3, we introduce a model of coupled oscillations of the suspended film and of the Plateau border, which predicts the experimental eigenfrequencies with no fitting parameter. In Sec. 4, we extend our setup to study the forced response of the suspended film and of the Plateau border to a sound field, and we measure resonance curves of the suspended film. We discuss this resonance in Sec. 5 through a simple extension of the previous model. In particular, we show the importance of the coupling between the film and the Plateau border.

\section{Free oscillations: experiments}

\subsection{Setup}

The axisymmetric system we study (sketched on figure 1) is a diaboloid, composed of three films: a horizontal circular one [denoted (f)] connected, via two catenary films [denoted $\left(c_{u}\right)$ and $\left(c_{l}\right)$ ] to two aluminum rings $(21 \mathrm{~mm}$ in diameter, one above and one below) separated by a distance $h$. The three films meet at a circular meniscus, also called Plateau border. This setup is adapted from our recent study on soap film extension (Seiwert et al., 2013). To create the diaboloid, the solid rings are first placed in close proximity to each other $(h \approx 0.5 \mathrm{~mm})$ and dipped in the foaming solution made of 10 $\mathrm{g} / \mathrm{L}$ sodium dodecyl sulfate (SDS) in water or water/glycerol mixtures. This creates a single (horizontal) film attached to both of them. The upper and lower suspending films appear naturally when the rings are separated to reach their final position ( $h$ varies between $2.8 \mathrm{~mm}$ and $6.3 \mathrm{~mm}$ depending on the experiment).

A key parameter of our system is the cross-sectional area of the Plateau border. We 
measure it by imaging the system from the side (Fig. 2a), and fitting the external profile (the rim) of the Plateau border. The fit takes into account both axisymmetry, surface tension and gravity, and it allows us to determine the cross-sectional area of the Plateau border $s_{P}$, which measures the Plateau border size. The fitting procedure leads to a relative uncertainty on $s_{P}$ of at most $6 \%$. This relative uncertainty is obtained by comparing the fitted cross section to the following cruder estimation. The rim of the Plateau border is fitted by an arc of circle of radius $R_{P}$, which would be its shape in the absence of gravity; the cross section of the Plateau border would then equal $(\sqrt{3}-\pi / 2) R_{P}^{2}$ (Dollet $\&$ Raufaste, 2014). We have checked that the relative difference between this crude estimate and the more complete fit does not exceed $6 \%$. The method used to prepare the system creates a "thick" Plateau border of cross-sectional area $s_{P} \simeq 0.6 \mathrm{~mm}^{2}$. It can be adjusted down to a minimal cross-sectional area $s_{P} \simeq 0.05 \mathrm{~mm}^{2}$, although not in a finely controllable way, by sucking out some of its liquid with a paper tissue.

Under the action of the capillary suction at the Plateau border, the central film is constantly draining throughout the experiment. By waiting at least one minute between film formation and the experiment itself, we ensure that the thinning of the film has slowed down enough to be negligible (less than $5 \%$ variation in thickness over the time needed to perform the measurements, typically $10 \mathrm{~s}$ ). We also systematically measure its thickness with a reflectometer just prior to starting the experiment.

We performed two different types of experiments. First, in free oscillations experiments, we perturb the position of the Plateau border and observe it as it returns towards equilibrium, gathering data on the fundamental eigenfrequency and the damping of the system. Second, in forced oscillations experiments, a sinusoidal sound wave is used to excite the system at a given frequency. We will return to forced oscillations from Sec. 4 onwards; let us first focus on free oscillations.

To generate the perturbation, we first create the three films with a distance $h=6.3$ $\mathrm{mm}$ between the rings, then suddenly raise the lower ring with a motorised stage (in approximately $25 \mathrm{~ms}$ ) until $h=2.8 \mathrm{~mm}$ or $h=4.8 \mathrm{~mm}$. The inertia of the Plateau border ensures that it does not move significantly over this short period of time, thus just after ring displacement, it rests with negligible velocity, approximately $1.75 \mathrm{~mm}$ away from its new equilibrium position. We image the system from the side with a high speed camera (at 100-500 frames per second, a typical image is shown in figure 2a), and track the position of the upper and lower boundaries of the Plateau border as a function of time.

As shown in the example of figure $2 \mathrm{~b}$, both boundaries move in phase with the same amplitude. They oscillate around their new equilibrium positions, and come to rest after a few oscillations. Except at short times, the movement is well fitted by an exponentially decaying sinusoid of the form

$$
z=z_{0}+A_{0} \mathrm{e}^{-2 \pi l f_{0} t} \sin \left(2 \pi f_{0} \sqrt{1-l^{2}} t\right),
$$

which is the expression of the unforced damped oscillations of an underdamped harmonic oscillator of eigenfrequency $f_{0}$ and damping coefficient $l$. In particular, the period remains constant within $10 \%$. The damping coefficient and the eigenfrequency of these free oscillations are found by fitting the data, and characterise each experiment. When analysing the data, we fit independently the upper and the lower boundaries, and plot their mean value (with error bars spanning the entire range).

In order to validate the setup, we first performed a series of test experiments to check that $f_{0}$ and $l$ do not depend on the method used to drive the Plateau border out of equilibrium. In particular, we tested two different displacement speeds for the ring, and 
(a)

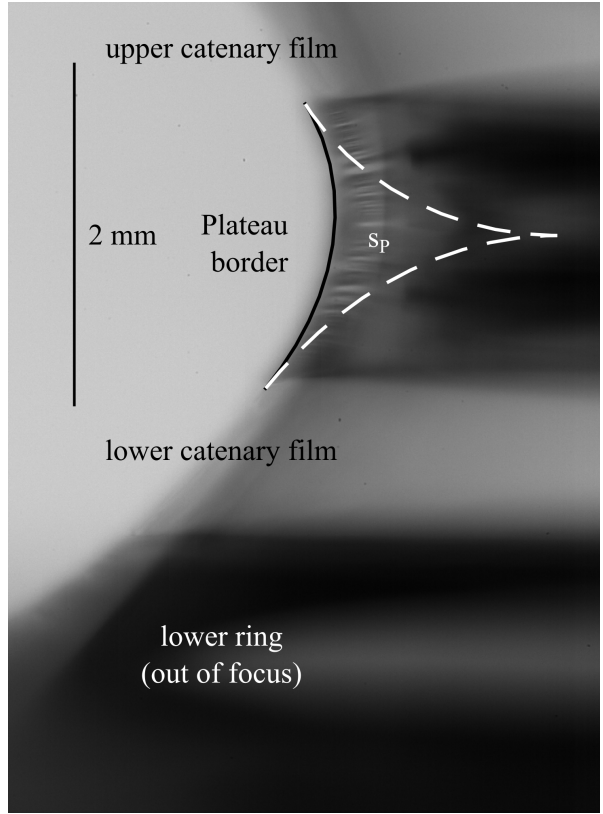

(b)

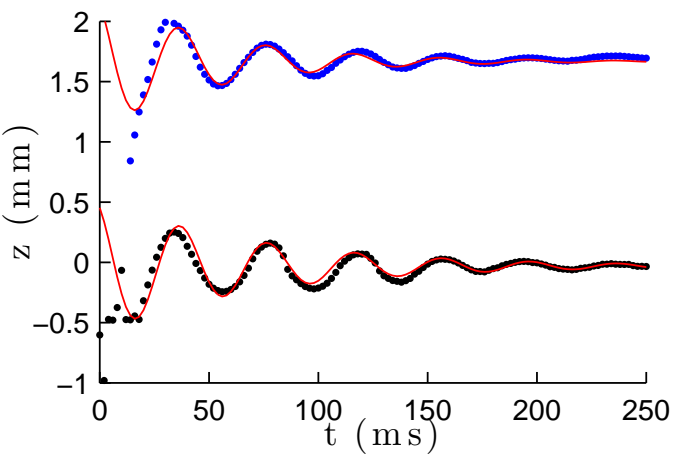

Figure 2. (a): Typical profile image of the Plateau border, from which we extract its radius (by fitting), and the position of its upper and lower boundaries. (b): Position of the upper and lower boundaries of the Plateau border, as a function of time, during a free oscillation experiment (with $h=4.8 \mathrm{~mm}$ and $s_{P}=0.4 \mathrm{~mm}^{2}$ ). Solid lines represent best fits of the form (2.1): $z(t)=z_{0}+A_{0} e^{-2 \pi l f_{0} t} \sin \left(2 \pi f_{0} \sqrt{1-l^{2}} t\right)$ with best fitting parameters $f_{0}=25 \mathrm{~Hz}$ and $l=0.12$ (upper) and $l=0.087$ (lower).

found no difference except for a short transient. We also checked that the film remains flat in these experiments, and follows the Plateau border.

\subsection{Results}

We plot in figure $3 \mathrm{a}$ the eigenfrequency $f_{0}$ as a function of the Plateau border crosssectional area $s_{P}$. It varies between 10 and $50 \mathrm{~Hz}$, and decreases with $s_{P}$. Additionally, $f_{0}$ increases when the gap between the rings goes from $h=4.8 \mathrm{~mm}$ (solid symbols) to $h=2.8 \mathrm{~mm}$ (asterisks). The damping coefficient $l$ is shown in figure $3 \mathrm{~b}$. The data are more scattered, because the fit for $l$ is more sensitive to experimental errors (especially in the tracking of the Plateau border). However, there is no systematic variation of $l$ with $s_{P}$.

We also tested different foaming solutions, all containing $10 \mathrm{~g} / \mathrm{L}$ of SDS dissolved in a $0 \%, 50 \%$ or $80 \%$ glycerol/water mixture. The main difference between these three solutions is their shear viscosity $\eta$, which goes roughly from $1 \mathrm{mPa} \cdot \mathrm{s}$ to $40 \mathrm{mPa} \cdot \mathrm{s}$ (see table 1). They also show a small difference in their density $\rho$. This large increase in shear viscosity, however, does not translate into an increase in the damping coefficient (figure $3 \mathrm{~b}$ ). 
(a)

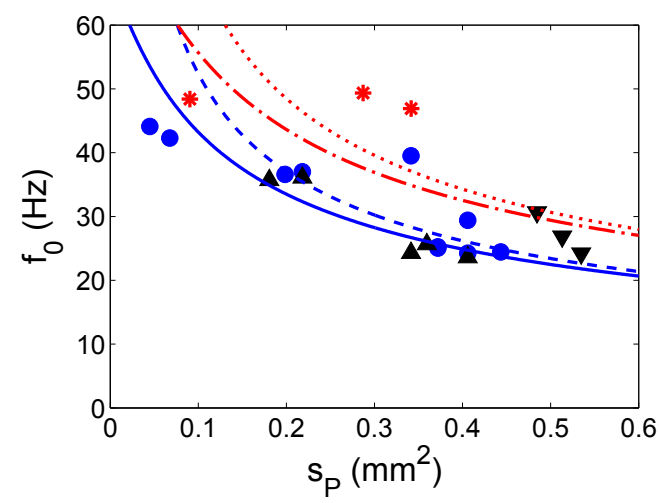

(b)

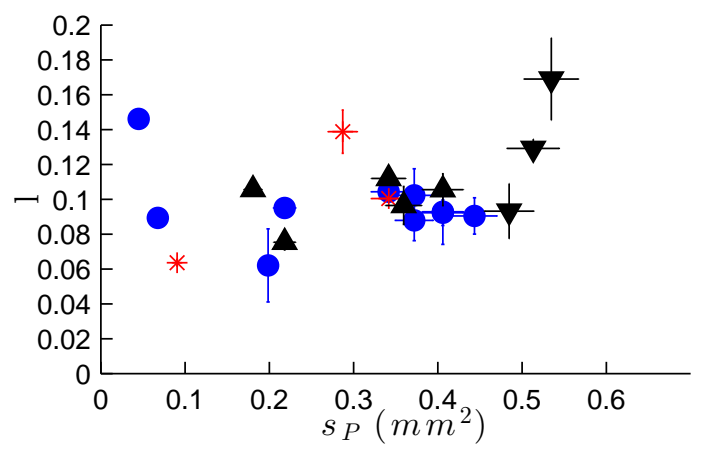

Figure 3. (a): Eigenfrequency $f_{0}$ and (b): damping coefficient $l$ measured by fitting free oscillations of the Plateau border, as a function of its cross-sectional area $s_{P}$. Asterisks are for $h=2.8 \mathrm{~mm}$, all other symbols are for $h=4.8 \mathrm{~mm}$. Asterisks and circles are for solution S (10 g/L SDS in water), upward and downward triangles are for solutions S50 and S80 containing respectively $50 \%$ and $80 \%$ of glycerol. For each experiment, the upper and lower boundaries are fitted independently, as illustrated in Fig. 2b, and error bars span the two extracted values. The plain and dashed lines are respectively the predictions (with no fitting parameter) for the eigenfrequency $\Omega_{P} / 2 \pi$ with $\Omega_{P}$ given by (3.4) of the Plateau border, and the fundamental frequency of the coupled system (see Sec. 3.4), for $h=4.8 \mathrm{~mm}$. Similarly, the dash-dotted and dotted lines are respectively the predictions for the eigenfrequency $\Omega_{P} / 2 \pi$ with $\Omega_{P}$ given by (3.4) of the Plateau border, and the fundamental frequency of the coupled system, for $h=2.8 \mathrm{~mm}$.

\begin{tabular}{|c|c|c|c|c|} 
solution & glycerol & surfactant & viscosity $\eta$ & density $\rho \mid$ \\
\hline S & 0 vol. \% & SDS (10 g/L) & $0.98 \pm 0.03 \mathrm{mPa} \cdot \mathrm{s}$ & 1.00 \\
S50 & 50 vol. \% & SDS (10 g/L) & $4.63 \pm 0.04 \mathrm{mPa} \cdot \mathrm{s}$ & 1.10 \\
S80 & 80 vol. $\%$ & SDS (10 g/L) & $38 \pm 2 \mathrm{mPa} \cdot \mathrm{s}$ & 1.16
\end{tabular}

TABLE 1. Foaming solutions used in the experiments, and their measured physical properties. The surface tension $\gamma=32 \mathrm{mN} / \mathrm{m}$ is the same for all solutions.

\section{Free oscillations: interpretation}

\subsection{Simplifying assumptions}

The upper and lower suspending films impose the equilibrium position of the Plateau border, and for small displacements around its equilibrium position, we expect that they act as a capillary spring. Together with the mass of the Plateau border and the film, this sets an eigenfrequency.

To further develop and evaluate this idea, let us write explicitly the balance between inertia and capillarity, which allows us to predict with no fitting parameter the observed eigenfrequency (as seen on figure 3a). In particular, we show in the next section that the dynamics of the film has to be taken into account. Our model is based on several approximations :

(a) The problem is purely axisymmetric.

(b) The thickness $e$ of the suspended film remains uniform at all times.

(c) The Plateau border is a rigid ring of radius $a$ and of cross-sectional area $s_{P}$ much smaller than $a^{2}$. The mass per unit length of this ring is $\mu=\rho s_{P}$. Moreover, its weight 
is neglected, so that the only forces acting on it are the capillary tractions of the three films.

(d) The oscillations of the Plateau border and the film are much smaller than the separation between the rings (and are treated at first order).

(e) The curvature of the suspending films in the diametral plane is negligible (figure 4). Hence, the capillary equilibrium imposes a $120^{\circ}$ angle between the suspending films, yielding the following geometrical relation between the film radius $b$ of the supporting rings, the radius $a$ of the Plateau border, and the gap $h$ between the two supporting rings: $b=a+h / 2 \sqrt{3}$.

$(f)$ All forms of dissipation (viscous dissipation in the liquid, in the air, thermal dissipation in the air, radiation damping) are neglected.

We now discuss the validity of these approximations. Approximation $(a)$ is validated by analysing experimental movies, and indeed no deviation from axisymmetry, in the film or in the Plateau border, is observed during free oscillations.

Assumption $(b)$ is also validated by direct visualisation of the system, on a specific set of validating experiments, with a slightly different imaging set-up than what is shown here. In order to observe the evolution of the thickness of the suspended film, it is illuminated by a monochromatic light, which reflects on both interfaces of the film and produces an interference pattern, as seen on figure 1b. Imaging the film from above thus gives us a time-resolved map of the relative thickness of the film. There are some local thickness heterogeneities, but we checked that there are no large-scale thickness gradients.

For the Plateau border to be represented by a ring of negligible cross-sectional area (approximation $(c)$ ), its characteristic length scale $\sqrt{s_{P}}$ must remain negligible compared to both the radius $a$ of the film and the distance between rings $h$. In our experiments, $\sqrt{s_{P}}$ reaches at most $1 \mathrm{~mm}$, while $a \approx 8 \mathrm{~mm}$ and $h \approx 5 \mathrm{~mm}$ or $3 \mathrm{~mm}$. The condition $\sqrt{s_{P}} \ll a$ is thus satisfied to within $12 \%$. The condition $\sqrt{s_{P}} \ll h$ is more questionable, particularly for the lowest distance between rings. However, the finite extension of the Plateau border is known not to modify the orientation of the films (apart from its weight, which we discuss next), a result known as the decoration theorem (Weaire \& Hutzler, 1999). Therefore, the forces exerted by the films should remain independent of the crosssectional area of the Plateau border, even if $\sqrt{s_{P}} / h$ is not particularly small.

Neglecting the weight of the Plateau border may seem questionable, as seen in figure 2a: the main effect of gravity is to dissymetrise the equilibrium position of the system, and the upper film is slightly more vertical than the lower one. To assess this approximation in terms of force balance, we must compare the weight per unit length $\mu g$ to the tractions per unit length $2 \sigma$ exerted by each of the films. In our experiments, $s_{P}$ is below $0.6 \mathrm{~mm}^{2}$, hence $\mu g / 2 \sigma$ remains below 0.1 . Taking into account the finite weight of the Plateau border is in fact straightforward. We have checked that including it in the force balance presented in Sec. 3.2 modifies the eigenfrequency by less than $6 \%$, which is comparable to the experimental uncertainties. Hence, we neglect weight for simplicity.

Approximation $(d)$ is easily justified: the amplitudes of the oscillations of the Plateau border (typically $0.5 \mathrm{~mm}$ ) and the film (at most $0.1 \mathrm{~mm}$ ) are ten times smaller than the distance between rings $(h \approx 5 \mathrm{~mm})$.

Approximation $(e)$ is mainly imposed for convenience: taking into account the curvature of the suspending films (whose shape is a catenary, since their total curvature must be nil) is tedious but straightforward on a static diaboloid (Salkin et al., 2014). Comparing the exact (curved) shape of a static diaboloid and the shape without curvature (in dashed line in Fig. 4), we have calculated that our approximation leads to a slight overestimation of the film radius, by at most $5 \%$.

Lastly, assumption $(f)$ directly follows from the small damping ratio $(l \approx 0.1)$ of the 


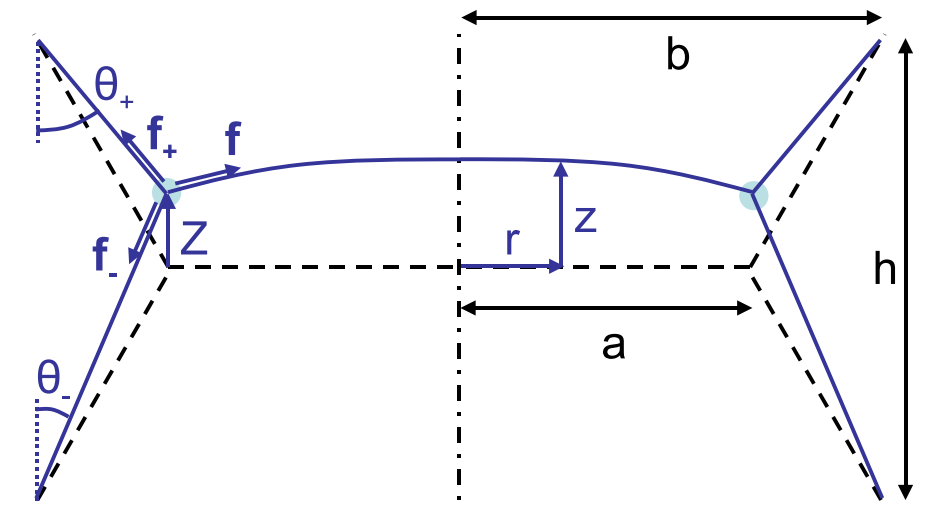

FIgURE 4. Sketch of the films in a diametral plane (not to scale). The dash-dotted line is the axis of revolution of the system. The dashed lines represent the assumed equilibrium configuration, see Sec. 3.1. The Plateau border is sketched as the blue circle at the junction of the films. Its motion is forced by the tractions $\mathbf{f}_{+}, \mathbf{f}_{-}$and $\mathbf{f}$ of the three films to which it is connected, respectively the upper suspending film, the lower suspending film, and the suspended film.

system. For small $l$, the damping affects the eigenfrequency by a factor of order $\ell^{2}$ (Landau \& Lifchitz, 1969). Hence, we may safely assume the eigenfrequency to be almost independent from the exact value of the dissipation. Of course, this completely overlooks the microscopic mechanisms at the origin of the observed damping, which are discussed in Appendix A.

\subsection{Dynamics of the Plateau border}

Let $z(r, t)$ be the vertical position of the films, and $Z(t) \equiv z(a, t)$ the vertical position of the Plateau border with respect to the equilibrium position $z=0$ (figure 4 ).

The motion of the Plateau border is driven by the capillary traction of the three films to which it is connected: each of these films exerts a force directed tangentially to the films (figure 4), and of magnitude $2 \sigma$. This comes from the fact that a given film is bounded by two air/liquid interfaces, with a surface tension $\sigma$ on each interface. Hence, the projection along the upwards vertical direction (henceforth the vertical projection) of the force per unit length exerted by the upper and lower suspending films is respectively: $f_{+z}=2 \sigma \cos \theta_{+}$and $f_{-z}=-2 \sigma \cos \theta_{-}$, where the projection angles $\theta_{ \pm}$are drawn in figure 4. Moreover, consistently with the assumption $(d)$ of small oscillations, the vertical slope of the suspended film is such that $\left|\partial_{r} z\right| \ll 1$. Hence, the vertical projection of the force per unit length exerted by the suspended film on the Plateau border is $f_{z}=$ $-2 \sigma \partial_{r} z(a, t)$. Summing these three forces and projecting vertically Newton's law on a unit length of Plateau border of mass $\mu$, we get

$$
\mu \ddot{Z}=f_{z}+f_{+z}+f_{-z}=-2 \sigma \partial_{r} z(a, t)+2 \sigma\left(\cos \theta_{+}-\cos \theta_{-}\right) .
$$

Assumption $e$ imposes that the suspending films projected in a diametral plane are straight lines (figure 4). Hence, from simple geometry, we get

$$
\cos \theta_{ \pm}=\frac{h / 2 \mp Z}{\left[(h / 2 \sqrt{3})^{2}+(h / 2 \mp Z)^{2}\right]^{1 / 2}} .
$$

Moreover, from assumption $(d),|Z| \ll h$, hence to first order in $|Z| / h$ :

$$
\cos \theta_{ \pm} \simeq \frac{\sqrt{3}}{2}\left(1 \mp \frac{Z}{2 h}\right)
$$


Using this equation to substitute for $\cos \theta_{ \pm}$in (3.1), we obtain

$$
\mu \ddot{Z}=-2 \sigma \partial_{r} z(a, t)-\frac{\sigma Z \sqrt{3}}{h} .
$$

The latter term corresponds to the forces exerted by the suspending films, which act as an effective spring. Equation (3.2) can be rewritten

$$
\ddot{Z}+\Omega_{P}^{2} Z=-\frac{2 \sigma}{\mu} \partial_{r} z(a, t),
$$

where the parameter $\Omega_{P}$ obeys

$$
\Omega_{P}^{2}=\frac{\sigma \sqrt{3}}{\mu h}=\frac{\sigma \sqrt{3}}{\rho s_{P} h} .
$$

Equation (3.3) is the equation of an undamped oscillator, coupled to the motion of the suspending film. The frequency $\Omega_{P} / 2 \pi$ is a decreasing function of the gap $h$ between the two rings, and of the cross-sectional area of the Plateau border: decreasing the gap shortens the suspending films, and thus stiffens their elastic response. It may come as a surprise that $\Omega_{P}$ does not depend on the radius of the suspended film $a$, since the mass of the Plateau border $2 \pi a \mu$ does scale like $a$. But the capillary force exerted by the suspending films also scales like $a$, and both contributions cancel out (this is somewhat hidden in our model by the fact that we write the equations per unit length of the Plateau border).

The frequency $\Omega_{P} / 2 \pi$ compares favorably with the measurements presented in figure $3 \mathrm{a}$ (dashed lines), with no adjustable parameters. However, it systematically overestimates the eigenfrequencies measured for the smallest sections $\left(s_{P}<0.1 \mathrm{~mm}^{2}\right)$. This suggests that the coupling with the oscillation of the film, represented by the right-hand side of (3.3) may not be neglected.

\subsection{Dynamics of the horizontal film}

To take into account the right-hand side of (3.3), the entire dynamics of the suspending film has to be modelled, since its precise shape enters that equation. The restoring force for the film is surface tension, and more precisely, when it deforms, its portion comprised between $r$ and $r+\mathrm{d} r$ is subjected to a vertical capillary force

$$
2 \pi(r+\mathrm{d} r) \times 2 \sigma \partial_{r} z(r+\mathrm{d} r, t)-2 \pi r \times 2 \sigma \partial_{r} z(r, t) \simeq 4 \pi \sigma \partial_{r}\left(r \partial_{r} z\right) \mathrm{d} r ;
$$

here $\left|\partial_{z} r\right| \ll 1$ is assumed, consistently with assumption $(d)$.

Moreover, several authors have shown that air inertia plays a major role on soap film vibrations (Couder, Chomaz \& Rabaud, 1989, Rutgers et al., 1996, Afenchenko et al., 1998, Kosgodagan Acharige, Elias \& Derec, 2014). Indeed, the film displaces a mass of air that scales like $\rho_{a} a^{3}$ (the radius of the film $a$ being the only relevant length scale, and $\rho_{a}$ being the density of air), whereas the mass of the film itself scales like $\rho e a^{2}$. The ratio of the two is thus $\rho_{a} a /(\rho e)$, and is of order 10 with $\rho \approx 10^{3} \mathrm{~kg} / \mathrm{m}^{3}, \rho_{a} \approx 1.2 \mathrm{~kg} / \mathrm{m}^{3}$, $a \approx 1 \mathrm{~cm}$ and $e \approx 1 \mu \mathrm{m}$, so that air inertia dominates that of the liquid contained within the film.

The effect of air inertia on film vibration has been modelled in detail by Joosten, 1984 and Sens, Marques \& Joanny, 1993. However, these studies considered films of infinite extension, well suited only to describe film vibrations with a sufficient number of radial modes (as in Kosgodagan Acharige, Elias \& Derec, 2014). We are here in the opposite case: we have checked that in the range of frequency currently discussed (below $200 \mathrm{~Hz}$ ), the film retains an approximately parabolic shape, and we cannot use these studies to 
derive the force exerted by the air. Taking its effect into account would thus require, in principle, to solve the complicated problem of the flow in the air for the precise geometry of our system. As an approximation, we neglect here both the influence of the suspending films and the rings, and that of the deformation of the suspended film, on the air motion. In this case, the effect of the air may be represented by an added mass corresponding to the motion of a rigid thin disk of radius $a$, which is $8 \rho_{a} a^{3} / 3$ (Lamb, 1932). In this ideal situation, the force per unit area on the disk is not uniform, but varies as $\left(a^{2}-r^{2}\right)^{-1 / 2}$. The divergence at the disk edge $r=a$ is due to the divergence of the velocity of the air flowing around the edge, and is certainly not present in our setup, given its geometry. For the sake of simplicity, we assume that the added mass is uniformly distributed on the suspended film. Hence, the portion of film comprised between $r$ and $r+\mathrm{d} r$ has an added mass $\left(8 \rho_{a} a^{3} / 3\right) \times\left(2 \pi r \mathrm{~d} r / \pi a^{2}\right)=16 \rho_{a} a r \mathrm{~d} r / 3$, in addition to its mass $2 \pi \rho e r \mathrm{~d} r$. Hence, applying Newton's law to this portion of film, accounting for the capillary force (3.5), the added mass force and the film inertia, we get

$$
\left(2 \pi \rho e r \mathrm{~d} r+\frac{16}{3} \rho_{a} a r \mathrm{~d} r\right) \partial_{t t} z=4 \pi \sigma \partial_{r}\left(r \partial_{r} z\right) \mathrm{d} r
$$

which can be rewritten

$$
\frac{1}{r} \partial_{r}\left(r \partial_{r} z\right)=\frac{1}{2 \sigma}\left(\rho e+\frac{8}{3 \pi} \rho_{a} a\right) \partial_{t t} z .
$$

The boundary conditions are

$$
\partial_{r} z(0, t)=0
$$

by symmetry, and the matching condition to the Plateau border

$$
z(a, t)=Z(t)
$$

Using the definition of the Fourier transform $\tilde{z}$ of $z$ :

$$
z(r, t)=\int_{-\infty}^{\infty} \tilde{z}(r, \omega) \mathrm{e}^{\mathrm{i} \omega t} \mathrm{~d} \omega
$$

and taking the Fourier transform of (3.7) gives

$$
\frac{1}{r} \partial_{r}\left(r \partial_{r} \tilde{z}\right)+q^{2} \tilde{z}=0
$$

with

$$
q^{2}=\frac{\left(8 \rho_{a} a / 3 \pi+\rho e\right) \omega^{2}}{2 \sigma} \equiv \frac{1}{a^{2}} \frac{\omega^{2}}{\omega_{f}^{2}}
$$

where

$$
\omega_{f}=\frac{1}{a} \sqrt{\frac{2 \sigma}{8 \rho_{a} a / 3 \pi+\rho e}} .
$$

Here $\omega_{f}$ is a typical angular frequency for the suspended film. With the experimental values $\sigma=0.03 \mathrm{~N} / \mathrm{m}, a=9 \mathrm{~mm}$ and $e=1 \mu \mathrm{m}$, we find $\omega_{f} \approx 43 \mathrm{~Hz}$.

Equation (3.10) can be rewritten in the form of a Bessel equation: $\partial_{\bar{r} \bar{r}} \tilde{z}+\partial_{\bar{r}} \tilde{z} / \bar{r}+\tilde{z}=0$, with $\bar{r}=q r$, whose general solution is a combination of the Bessel functions of order zero $J_{0}(q r)$ and $Y_{0}(q r)$. The latter is discarded here since it is singular at $r=0$. The boundary conditions $(3.8)$ and (3.9) give $\partial_{r} \tilde{z}(0, \omega)=0$, and $\tilde{z}(a, \omega)=\tilde{Z}(\omega)$, where $\tilde{Z}$ is the Fourier transform of $Z$. The solution of (3.10) obeying these two conditions is

$$
\tilde{z}(r, \omega)=\tilde{Z}(\omega) \frac{J_{0}(q r)}{J_{0}(q a)}=\tilde{Z}(\omega) \frac{J_{0}\left(r \omega / a \omega_{f}\right)}{J_{0}\left(\omega / \omega_{f}\right)},
$$


where $J_{0}$ is the Bessel function of the first kind of order zero. This function is known to describe the shape of the fundamental mode of vibration of circular soap films (see e.g. Kosgodagan Acharige, Elias \& Derec, 2014) and more generally of circular elastic membranes (see e.g. Chaigne \& Kergomard, 2008).

\subsection{Coupled dynamics}

We can now couple the dynamics of the film back to that of the Plateau border. Taking the Fourier transform of (3.3), we obtain

$$
\left(-\omega^{2}+\Omega_{P}^{2}\right) \tilde{Z}(\omega)=-\frac{2 \sigma}{\mu} \partial_{r} \tilde{z}(a, \omega) .
$$

From (3.13),

$$
\partial_{r} \tilde{z}(a, \omega)=-\frac{\omega}{a \omega_{f}} \tilde{Z}(\omega) \frac{J_{1}\left(\omega / \omega_{f}\right)}{J_{0}\left(\omega / \omega_{f}\right)} .
$$

Hence $\Delta \tilde{Z}=0$, with

$$
\Delta=\Omega_{P}^{2}-\omega^{2}-\omega_{P}^{2} \frac{\omega}{\omega_{f}} \frac{J_{1}\left(\omega / \omega_{f}\right)}{J_{0}\left(\omega / \omega_{f}\right)}
$$

with another angular frequency $\omega_{P}$ which obeys

$$
\omega_{P}^{2}=\frac{2 \sigma}{\mu a}=\frac{2 \sigma}{\rho s_{P} a},
$$

and is based on surface tension and the mass of the Plateau border. It does not scale like the real angular eigenfrequency of the Plateau border $\Omega_{P} ; \omega_{P}$ varies with the radius of the films $a$, whereas $\Omega_{P}$ varies with the gap $h$ between the rings.

The eigenfrequencies of the system are the zeros of $\Delta$ given by (3.14), which can be found numerically. There is an infinite number of solutions, corresponding to different modes of vibration for the films. We plot in figure $3 \mathrm{a}$ (solid lines) the lowest positive one (the fundamental frequency) as a function of $s_{P}$. This predicted eigenfrequency is lower than the eigenfrequency $\Omega_{P} / 2 \pi$ of the Plateau border alone (dashed lines), due to the coupling with the film, with a difference increasing for decreasing $s_{P}$. It fits the measured frequencies over the full range of $s_{P}$ with no adjustable parameters; in particular, for $h=4.8 \mathrm{~mm}$, it is much better than $\Omega_{P} / 2 \pi$ for the lower range of $s_{P}$. Again, the measured frequencies of free oscillations do not come only from the sole Plateau border, but are also sensitive to the coupling with the film, especially when the cross-sectional area of the Plateau border is small. For $h=2.8 \mathrm{~mm}$, the coupled model does not seem to better fit the experimental data than the uncoupled one (Fig. 3a). A possible reason is that assumption $(c)$ breaks down: the suspending films are then very short, and the Plateau border may start to interact with the rings.

To get a better insight on this coupling, an approximate but analytical solution of $\Delta=0$ can be found by expanding the Bessel's functions as Taylor series close to zero, and keeping only the lowest term: $\omega J_{1}\left(\omega / \omega_{f}\right) /\left[\omega_{f} J_{0}\left(\omega / \omega_{f}\right)\right] \simeq \omega^{2} / 2 \omega_{f}^{2}$. In the experimental range of frequencies of figure $3 \mathrm{a}, \omega / \omega_{f}$ remains lower than 1.1, and the series expansion remains accurate within $19 \%$. Then $(3.14)$ becomes: $0=\Omega_{P}^{2}-\omega^{2}-\omega_{P}^{2} \omega^{2} / \omega_{f}^{2}$. The analytical approximation $\Omega_{0}$ of the eigenfrequency is the solution of this equation, hence

$$
\Omega_{0}^{2}=\frac{\Omega_{P}^{2}}{1+\omega_{P}^{2} / \omega_{f}^{2}} .
$$

In this expression, $\Omega_{P}$ is the fundamental frequency (3.4) of the Plateau border when it is decoupled from the film. The ratio $\omega_{P}^{2} / \omega_{f}^{2}$ quantifies the coupling between the film 

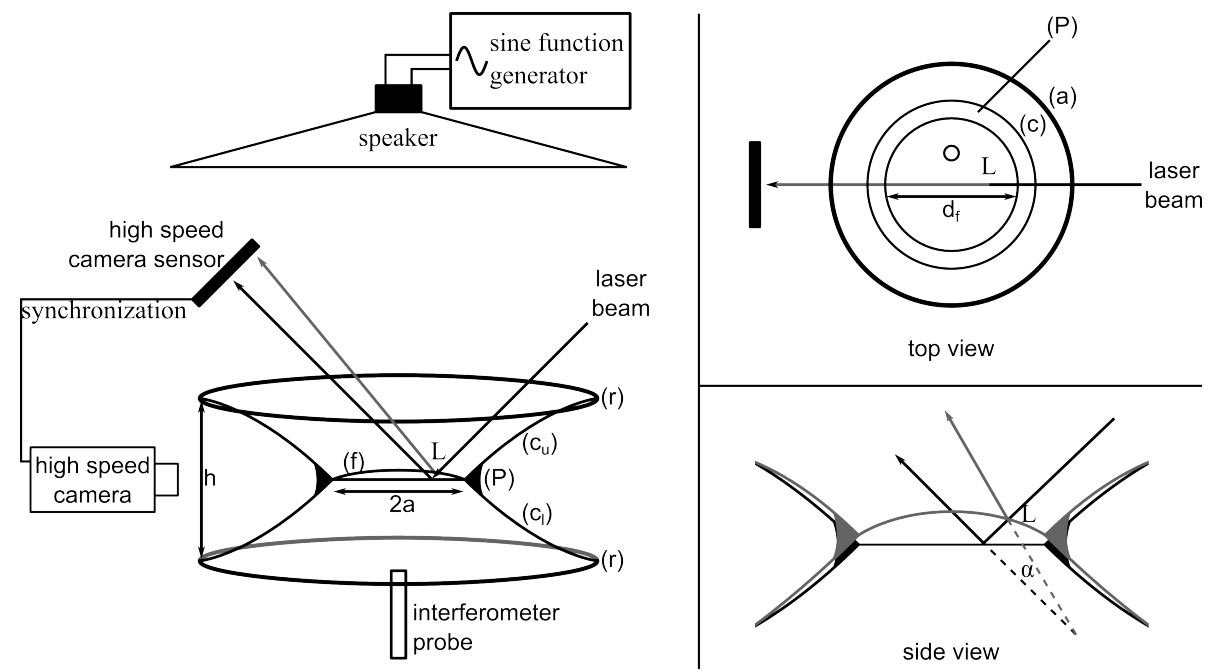

FiguRE 5. Experimental setup (not to scale) : the axisymmetric horizontal soap film of interest (f) is suspended between two aluminum rings (a) via catenary films (c). These films meet at the Plateau border (P) at an angle of approximately $120^{\circ}$. (P) is backlit and its profile is monitored by a high speed camera to track its position $z_{P}$. A laser beam is reflected off the film (f) at point $\mathrm{L}$, and tracking its position on the sensor of a second high speed camera (which is synchronised with the first) allows us to measure the local slope of the film $\alpha$ (with a precision of $1 \mathrm{mrad}$ ). Assuming that the film keeps a parabolic shape, this angle is easily related to the maximum deflection of the film (in the frame of the Plateau border) $z_{f}$.

and the Plateau border. After (3.15) and (3.12), it is inversely proportional to $s_{P}$, as is $\Omega_{P}^{2}$, see (3.4). Hence, the eigenfrequency tends towards a finite value at vanishing crosssectional area of the Plateau border, which is the eigenfrequency of the film itself (with "elastic" boundary conditions imposed by the suspending films).

To conclude this section, we must mention that we have no satisfactory theoretical explanation for the experimental value of the damping. A possible source of dissipation, coming from the viscous flow in the transition region between the films and the Plateau borders, is discussed in Appendix A.1. We show that while it gives a good order of magnitude for the solution without glycerol, it predicts that the damping ratio scales like $\eta^{2 / 3}$, in contradiction with our data.

\section{Forced oscillations: experiments}

\subsection{Setup}

Compared to free oscillations, the setup used to observe forced oscillations is more involved (figure 5). The system of three films is made as described previously from the $\mathrm{S}$ solution (SDS in pure water, see table 1) with a distance $h=4.8 \mathrm{~mm}$ between rings. A loudspeaker is fastened $50 \mathrm{~cm}$ above the film, facing down. It is driven by a function generator coupled to an amplifier, and used to generate purely sinusoidal sound waves of forcing frequencies $f$ ranging between $50 \mathrm{~Hz}$ and $200 \mathrm{~Hz}$, and intensities at the film up to $105 \mathrm{~dB}$. The intensity is checked with a sonometer, and adjusted if necessary within 1 $\mathrm{dB}$ prior to each experiment. The lower limit of the forcing frequency range correspond to the lowest attainable forcing frequency for our experiment, whereas above the upper limit the oscillations of the Plateau border become too small to be measured.

When sound waves excite the system, both the Plateau border and the film oscillate 
at the forcing frequency imposed by the speaker. We measure the amplitude of the oscillations of the Plateau border in the same way as for free oscillations but with a higher temporal resolution, up to 2000 frames per second. The movement of the film, however, cannot be measured with the same technique, as it is shadowed by the Plateau border when observed from the side.

Instead, a laser beam is reflected on its surface, $3.3 \mathrm{~mm}$ away from its centre, at point $\mathrm{L}$ (figure 5). The reflected beam is projected directly on the sensor of a second high speed camera (synchronised with the first one, to allow a measure of the phase difference between the film and the Plateau border) placed $10.1 \mathrm{~cm}$ from L, and perpendicular (within $5^{\circ}$ ) to the laser beam when the film is at rest. When the system oscillates, the spot produced by the laser moves across the sensor, due to three different mechanisms: 1) $\mathrm{L}$ moves in the vertical direction, due to the combined movements of the Plateau border and the film; 2) as the films moves in the vertical direction, it intersects the laser in a different spot (L moves along the radius of the film) and 3) the local slope of the film changes and deflects the laser from its original direction. In practice, in our experiments the latter is always dominant (the first two effects are approximately ten times weaker than the third one), and simple geometry allows us to correlate the position of the laser spot on the sensor to the local slope of the film in L. Given the range of excitation forcing frequencies tested in this work, the film features an axisymmetric deformation. We have checked that more complex, non-axisymmetric patterns, such as the ones shown in Couder, Chomaz \& Rabaud, 1989, Afenchenko et al., 1998, Vega, Higuera \& Weidman, 1998, Elias, Hutzler \& Ferreira, 2007, appear on the film only above $200 \mathrm{~Hz}$. This allows us to deduce, from this measure of a local slope, its entire profile, see equation (3.13) for the predicted axisymmetric profile. Moreover, to simplify the analysis of the data, we ideally want the direction of the laser and the radius of the film containing $\mathrm{L}$ do be in the same plane (see figure 5), so that the laser beam only moves in a vertical plane after it has been reflected off the film. We show on figure 6 a a typical result of the tracking of the laser spot on the sensor: in practice its movement is not a strict line, but rather a tilted ellipse. We checked that both the tilt and the eccentricity are quantitatively compatible with slight misalignments of the system (namely the fact that the plane containing the incident laser beam is slightly off the centre of the suspended film). We thus only use the vertical position of the spot to determine the local (axisymmetric) slope of the film in L.

Alternatively, we performed a series of qualitative observations of the film, without measuring its deflection. Instead, we used an (expanded) laser beam to generate an interference pattern on the entire film, which we imaged with a high speed camera. This helped us ensure that the thickness of the film remains constant throughout the experiment. In fact, redistribution of the liquid within the film (self-adaptation) does occur at certain forcing frequencies, as previously observed by Boudaoud, Couder \& Ben Amar, 1999, but over longer timescales than the duration of the experiment (which lasts typically $0.5 \mathrm{~s}$, starting 1 to 2 seconds after the speaker has been turned on).

Figure 7 shows a typical result for the movement of the Plateau border. Both its boundaries oscillate sinusoidally with a fixed amplitude, at the forcing frequency $f$. Sinusoidal fits allow us to extract this amplitude, as well as the phase of these oscillations. Their amplitudes and their phases, however may differ slightly (as seen on this particular example). This is the signature of complex deformations of the Plateau borders: in those cases, it is not simply translated as a whole and analysis of the experimental movies reveals waves travelling along the Plateau border. However, the amplitude of these deformations (typically $0.1 \mathrm{~mm}$ ) is smaller than the vertical translation of the Plateau border.

Figure $6 \mathrm{~b}$ shows the corresponding data for the film, namely its local slope at point $\mathrm{L}$, where the laser beam is reflected. Exactly like the Plateau border, the film oscillates 
(a)

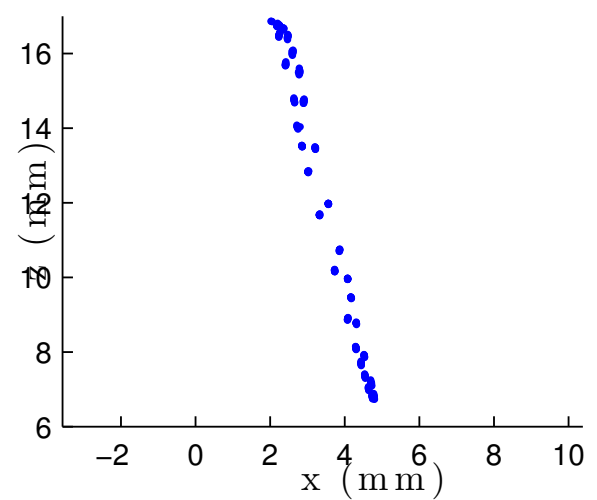

(b)

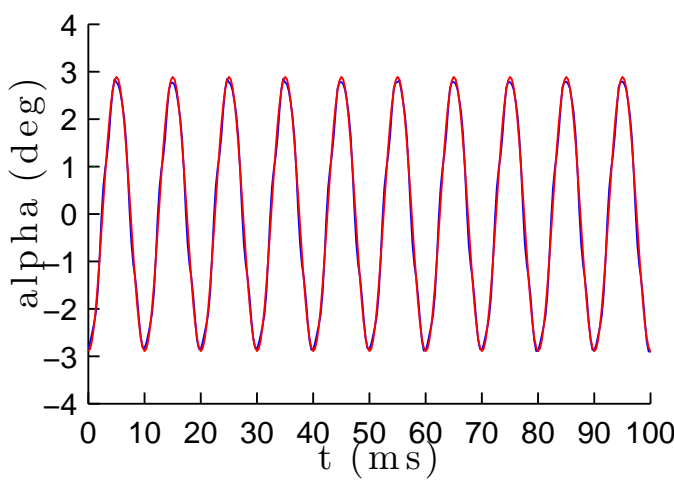

Figure 6. Observation of the film in a forced oscillation experiment with $f=100 \mathrm{~Hz}$, a sound amplitude of $90 \mathrm{~dB}$, and $s_{P}=0.48 \mathrm{~mm}^{2}$. (a): Successive positions of the laser spot on the sensor of the high-speed camera ( $x$ and $y$ are coordinates on the sensor itself, $y$ runs in the vertical direction). (b): Local slope of the film in L, as a function of time. The time base is the same as in figure 7. The solid line is a sinusoidal fit of the form $z=z_{0}+A \cos (2 \pi f t+\phi)$, with amplitude $A=2.9^{\circ}$ and phase $\phi=3.12 \mathrm{rad}$. For clarity, only a portion of the data is plotted (the entire set is $1.4 \mathrm{~s}$ long).

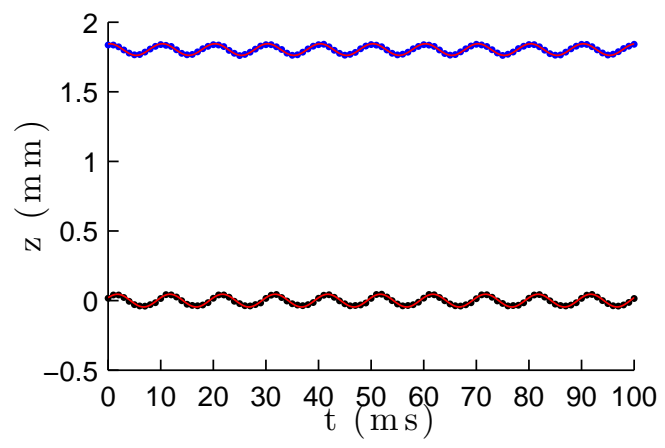

FIGURE 7. Vertical position of the upper and lower boundaries of the Plateau border in a forced oscillation experiment with $f=100 \mathrm{~Hz}$, a sound amplitude of $90 \mathrm{~dB}$ and $s_{P}=0.48 \mathrm{~mm}^{2}$. Solid lines represent sinusoidal fits of the form $z=z_{0}+A \cos (2 \pi f t+\phi)$, with amplitudes $A_{\text {sup }}=0.042 \mathrm{~mm}$ and $A_{\text {inf }}=0.046 \mathrm{~mm}$, and phases $\phi_{\text {sup }}=2.93 \mathrm{rad}$ and $\phi_{\text {inf }}=2.04 \mathrm{rad}$; notice the significant phase difference $\phi_{\text {sup }}-\phi_{\text {inf }}$. For clarity, only a portion of the data is plotted (the entire set is $1.4 \mathrm{~s}$ long.

with a given amplitude at the forcing frequency $f$, and fitting provides both its amplitude and its phase (in the same time base as the Plateau border). Given the diameter of the film and the position of $\mathrm{L}$, the maximum deflection $z_{f}$ of the film (at its centre, in the reference frame of the Plateau border) is then proportional to the the slope that we measure: $z_{f}(\mathrm{~mm}) \approx 0.16 \alpha\left(^{\circ}\right)$.

\subsection{Results}

Presenting the frequency-dependent amplitude of the forced oscillations of the Plateau border and the suspended film, and analysing these data, is not straightforward. Indeed, the coupling between the film and the Plateau border, hence the result of the experiments, depends strongly on the cross-sectional area of the Plateau border. Ideally, one would use the same three-film system to test a variety of forcing frequencies, to keep both the 
(a)
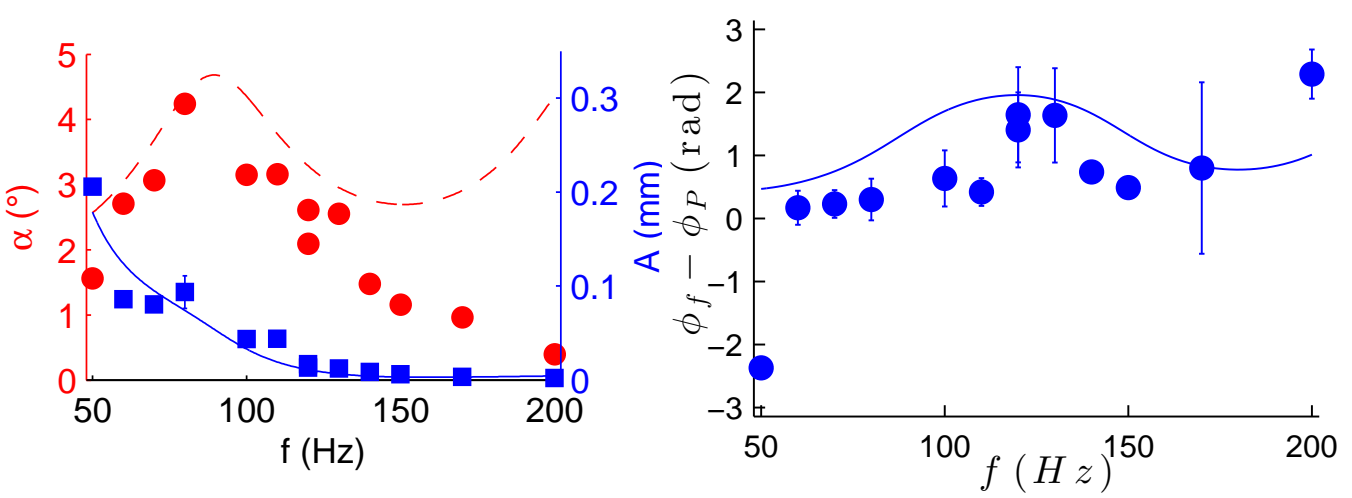

Figure 8. (a): Amplitude of the film (maximum angle $\alpha$ at point L, circles) and of the Plateau border (squares) as a function of the forcing frequency, for $h=4.8 \mathrm{~mm}$, a forcing amplitude of $90 \mathrm{~dB}$, and a cross section larger than $0.3 \mathrm{~mm}^{2}$. Lines are fit by the model (5.5)-(5.6), with $\bar{\lambda}=0.4$ : the plain curve is the prediction for $|\hat{Z}|$, and the dashed curve for $|\zeta|$. The lowest forcing frequency $(50 \mathrm{~Hz})$ is very close to the resonant frequency of our set-up, thus the corresponding data might be considered an arte fact. (b): Corresponding phase difference between the film and the Plateau border. The solid line is the prediction of the model.

film and the Plateau border exactly the same, but it is in practice impossible to achieve due the short lifetime of the films. Instead, each freshly prepared system is subjected to a single forcing frequency. An additional difficulty is then that the cross-sectional area of the Plateau border is impossible to control exactly (the thickness of the film also varies between experiments, up to a factor of three, but as mentioned earlier its mass is small compared to the added mass of the air, and we expect no significant effect associated with this variation). In fact, the cross-sectional areas of the Plateau borders are distributed fairly homogeneously across their entire range (the distribution is similar to that of the data of figure $3 \mathrm{a}$ ).

To simplify the representation of the data we separate, somewhat arbitrarily, our experiments in two categories: the ones with "large" Plateau borders (where $s_{P}>0.3 \mathrm{~mm}^{2}$, with an average for this series $\left\langle s_{P}\right\rangle=0.48 \mathrm{~mm}^{2}$, and a standard deviation of $0.11 \mathrm{~mm}^{2}$ ), and the ones with "small" Plateau borders $\left(s_{P}<0.2 \mathrm{~mm}^{2},\left\langle s_{P}\right\rangle=0.11 \mathrm{~mm}^{2}\right.$, and standard deviation $0.07 \mathrm{~mm}^{2}$ ). This choice also corresponds, loosely speaking, to a separation between a weak and a strong coupling between the films and the Plateau border, respectively for large and small Plateau borders (Sec. 3).

Starting with large Plateau borders, we show on figure 8a the response of the film and of the Plateau border as a function of the forcing frequency. The response of the film is typical of a resonance: it shows a maximum at $90 \mathrm{~Hz}$, with a 3 -dB bandwidth between 50 and $130 \mathrm{~Hz}$. The amplitude of the Plateau border is a decreasing function of the forcing frequency, with a small hump around $90 \mathrm{~Hz}$. The phase difference between the film and the Plateau border is displayed on figure 8b; except at $50 \mathrm{~Hz}$, it is positive, and increases from 0 to 2 rad between $60 \mathrm{~Hz}$ and $200 \mathrm{~Hz}$.

The case of small Plateau borders is shown on figure 9, and the response of the system is qualitatively different from figure $8 \mathrm{a}$. A resonance peak still appears, at a forcing frequency $110 \mathrm{~Hz}$ slightly larger than before; but now, the amplitude of the film decreases with the forcing frequency in the range 50 to $90 \mathrm{~Hz}$. The amplitude of the Plateau border is still a decreasing function of the forcing frequency, with again a small hump at $110 \mathrm{~Hz}$.

Qualitatively, these results fit with the picture of coupled oscillations. To elaborate, 
(a)

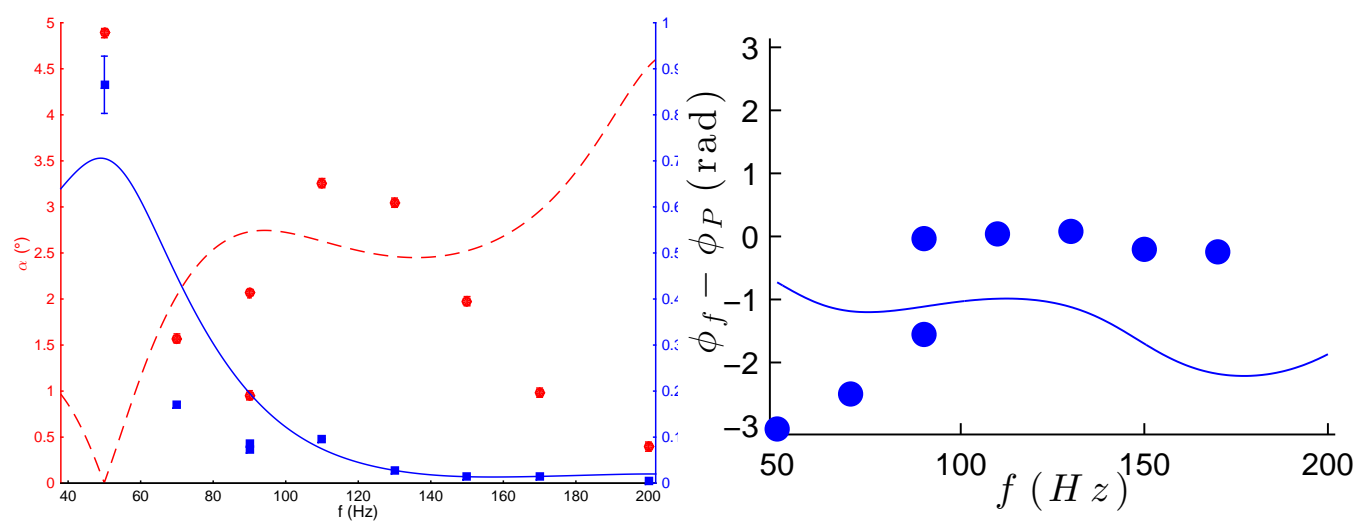

Figure 9. (a): Amplitude of the film (maximum angle $\alpha$ at point L, circles) and of the Plateau border (squares) as a function of the forcing frequency, for $h=4.8 \mathrm{~mm}$, a forcing amplitude of $90 \mathrm{~dB}$, and a cross section smaller than $0.3 \mathrm{~mm}^{2}$. Lines are fit by the model (5.5)-(5.6), with $\bar{\lambda}=0.4$ obtained from by fitting "large" Plateau borders: the plain curve is the prediction for $|\hat{Z}|$, and the dashed curve for $|\zeta|$. (b): Corresponding phase difference between the film and the Plateau border. The solid line is the prediction of the model.

for large Plateau borders, the resonance frequency of the film and the fundamental eigenfrequency of the Plateau border are well separated (the latter is below $30 \mathrm{~Hz}$ for $s_{P}>0.3 \mathrm{~mm}^{2}$, see figure $3 \mathrm{a}$ ), hence the resonance of the film is not influenced much by the dynamics of the Plateau border. For small Plateau borders, however, these two frequencies are closer, and the film and the Plateau border may display a stronger interaction for forcing frequencies between the two eigenfrequencies, which may result in a different response of the system. We develop this discussion in the next section, by adapting our model of Sec. 3 to the case of forced oscillations.

\section{Forced oscillations: modelling}

\subsection{Resonance frequency of the suspended film}

The model of Sec. 3 can already predict the resonance frequency of the film. Equation (3.13) shows that the amplitude of the film has singularities when $q a\left(\right.$ or $\left.\omega / \omega_{f}\right)$ is a zero of $J_{0}$. The lowest positive root of $J_{0}$ is approximately 2.405 , hence the lowest resonance frequency of the film equals $2.405 \omega_{f} / 2 \pi$, where $\omega_{f}$ is given by (3.12). This gives a value of $103 \mathrm{~Hz}$, in good agreement with the measured resonance frequency (figures $8 \mathrm{a}$ and 9, respectively $90 \mathrm{~Hz}$ and $110 \mathrm{~Hz}$ ). Such a good agreement is remarkable (and perhaps fortuitous), because this eigenfrequency crucially depends on the air inertia, which we modeled quite crudely as the added mass of a rigid disk in Sec. 3.3. It does confirm, however, the validity and the robustness of the assumptions made.

\subsection{Coupled dynamics and forcing}

We now include the effect of the forcing sound waves in our model. Since the speed of sound in air is $340 \mathrm{~m} / \mathrm{s}$ and the highest forcing frequency is $200 \mathrm{~Hz}$, the acoustic wavelength is at least $1.7 \mathrm{~m}$ in our experiments, much larger than the size of the system, hence air flow around the films can be taken as incompressible. For simplicity, we further assume that the pressure is uniform on each side of the suspended film, and we denote the pressure difference between the lower and upper side $\Delta P(t)=\Delta P_{0} \cos \omega t$. This 
disregards the complexity of the pressure field around the system; but our goal is not to solve the complicated air flow around the films, but to model the acoustic forcing as simply as possible. We keep assumption (e) of Sec. 3.1, which implies that we neglect the influence of the pressure difference on the suspending films. We also neglect its influence of the Plateau border, consistently with the assumption that $s_{P} \ll a^{2}$. Hence, we add in the right-hand side of the force balance (3.6) of the portion of film comprised between $r$ and $r+\mathrm{d} r$ the term $2 \pi r \Delta P(t) \mathrm{d} r$, while the force balance of the Plateau border (3.3) is unaffected.

However, the resonant curved of figures $8 \mathrm{a}$ and 9 show that the damping cannot be neglected to predict the response of the system: this is the only assumption of our model that we need to reconsider. Because we could not determine the real source of the dissipation in our system, we chose the simplest possible form and use a simple linear damping force proportional to the vertical velocity of the film, $\partial_{t} z(r, t)$ : we plug in the right-hand side of (3.6) a term equal to $-2 \pi r \mathrm{~d} r \times \lambda \partial_{t} z$, where $\lambda$ is a phenomenological damping coefficient. With this damping term and the pressure contribution, (3.6) becomes

$$
\left(2 \pi \rho e r \mathrm{~d} r+\frac{16}{3} \rho_{a} a r \mathrm{~d} r\right) \partial_{t t} z=4 \pi \sigma \partial_{r}\left(r \partial_{r} z\right) \mathrm{d} r+2 \pi r \Delta P(t) \mathrm{d} r-2 \pi \lambda r \partial_{t} z \mathrm{~d} r
$$

and (3.7) becomes

$$
\frac{1}{r} \partial_{r}\left(r \partial_{r} z\right)+\frac{\Delta P(t)}{2 \sigma}-\frac{\lambda}{2 \sigma} \partial_{t} z=\frac{1}{2 \sigma}\left(\rho e+\frac{8}{3 \pi} \rho_{a} a\right) \partial_{t t} z .
$$

Therefore, writing $z(r, t)=\operatorname{Re}\left[\hat{z}(r) \mathrm{e}^{-\mathrm{i} \omega t}\right]$ and $Z(t)=\operatorname{Re}\left(\hat{Z} \mathrm{e}^{-\mathrm{i} \omega t}\right)$, (3.6) we have instead of (3.10)

$$
\frac{1}{r} \frac{\mathrm{d}}{\mathrm{d} r}\left(r \frac{\mathrm{d} \hat{z}}{\mathrm{~d} r}\right)+q_{\lambda}^{2} \hat{z}=\frac{\Delta P_{0}}{2 \sigma}
$$

with $q_{\lambda}^{2}=q^{2}(1-\mathrm{i} \bar{\lambda})$ and the dimensionless film damping coefficient $\bar{\lambda}=\lambda \omega / 2 \sigma q^{2}$, while the boundary conditions (3.8) and (3.9) give: $\mathrm{d}_{r} \hat{z}(0)=0$, and $\hat{z}(a)=\hat{Z}$. Solving (5.2) gives

$$
\hat{z}=A J_{0}\left(q_{\lambda} r\right)+\frac{\Delta P_{0}}{2 \sigma q_{\lambda}^{2}} .
$$

Hence, if we quantify the film deformation by the length $\zeta=\hat{z}(0)-\hat{Z}$ (the maximum deflection of the film, in the frame of the Plateau border), we have

$$
\hat{z}(r)=\hat{Z}+\frac{J_{0}\left(q_{\lambda} r\right)-J_{0}\left(q_{\lambda} a\right)}{1-J_{0}\left(q_{\lambda} a\right)} \zeta
$$

hence

$$
\frac{\mathrm{d} \hat{z}}{\mathrm{~d} r}=\frac{q_{\lambda} J_{1}\left(q_{\lambda} r\right)}{1-J_{0}\left(q_{\lambda} a\right)} \zeta,
$$

showing that the slope of the film is proportional to $\zeta$ and independent of the motion of the Plateau border, which is convenient to compare with the experiments. Inserting (5.3) in (5.2), we get

$$
\hat{Z}-\frac{J_{0}\left(q_{\lambda} a\right)}{1-J_{0}\left(q_{\lambda} a\right)} \zeta=\frac{\Delta P_{0}}{2 \sigma q_{\lambda}^{2}}
$$

From (5.4), we compute

$$
\mathrm{d}_{r} \hat{z}(a)=\frac{q_{\lambda} J_{1}\left(q_{\lambda} a\right) \zeta}{1-J_{0}\left(q_{\lambda} a\right)} .
$$


Inserting in (3.3) and using the definition (3.15) of $\omega_{P}$, we get

$$
\left(-\omega^{2}+\Omega_{P}^{2}\right) \hat{Z}=-\omega_{P}^{2} \frac{q_{\lambda} a J_{1}\left(q_{\lambda} a\right)}{1-J_{0}\left(q_{\lambda} a\right)} \zeta .
$$

The solution of the linear system of Eqs. (5.5) and (5.6) provides a prediction of the forced coupled oscillations: the amplitude of the Plateau border oscillations is $|\hat{Z}|$, the slope of the film is proportional to $|\zeta|$, and the phase difference between the film and the Plateau border is $\arg \zeta-\arg \hat{Z}$. We plot the comparison of the predictions of the model and the experiments for large Plateau borders in figure 8, up to a constant prefactor, treating the dimensionless film damping coefficient as a fitting parameter, and using as an input the average cross-sectional area $\left\langle s_{P}\right\rangle=0.48 \mathrm{~mm}^{2}$. Concerning the amplitudes, a good agreement is found for a damping coefficient $\bar{\lambda}=0.4$ (figure 8a). The model predicts very well the position of the resonance. Notice that the resonance frequency is not a fitting parameter. The agreement between the experiment and the model is correct for the phase difference (figure 8b). This confirms that our model of the masses and stiffnesses involved in the system is quite satisfactory.

On the other hand, our simple model breaks down above $150 \mathrm{~Hz}$, where it predicts the wrong variation for the amplitude of the film oscillations. The most probable cause for this discrepancy is the simplistic dissipative term: a tailored (and arbitrary) dissipation increasing with the forcing frequency, i.e. a dispersive dissipation (e.g. with a frequencydependent damping coefficient $\lambda$ of the form $\lambda \propto \omega^{2}$ ) does allow us to practically collapse our model to the high forcing frequency part of the data, while maintaining a good fit around the resonance. As another possible reason for this discrepancy, it turns out that the assumption $(c)$ made in Sec. 3.1, of a rigid Plateau border, breaks down in this range of forcing frequencies. Indeed, as already mentioned in Sec. 4.1, the Plateau border displays strong deformations, with a significant phase difference between its upper and lower boundaries, that increases at increasing forcing frequency (data not shown). This echoes recent observations of strong Plateau border distortions, with modulations of cross-sections (Elias et al., 2014, Cohen et al., 2014).

We now compare the experiments and the model for small Plateau borders, using as an input the average cross-sectional area $\left\langle s_{P}\right\rangle=0.11 \mathrm{~mm}^{2}$, and $\bar{\lambda}=0.4$. The agreement on the amplitudes is only qualitative (figure 9): the model does reproduce the strong coupling, with a double resonance peak for the film amplitude, but the first peak is seen at forcing frequencies significantly lower than in experiments. Note that the comparison is more difficult to make in this case, because the values of $s_{P}$ are more dispersed (the ratio of the standard deviation to the mean of $s_{P}$ equals $59 \%$ for these small Plateau borders, whereas it is $22 \%$ for the big ones).

As in the case of free oscillations, we could not pinpoint with certainty the source of the dissipative term. Amongst the different possibilities that we explored, the most relevant is the viscous dissipation in the air that is displaced by the film. To estimate it, we represented the film by a disk of radius $a$, in coherence with our estimate for the added mass of the film (details are given in Appendix A.2). However, the predicted damping is around five times smaller than what is observed experimentally. This may be due to the crudeness of our approximation, or to the fact that another source of dissipation is dominant. We have also checked that in the range of forcing frequencies of the forced oscillation experiments, the damping of the Plateau border, which could be added as a term proportional to $\dot{Z}$ in the l.h.s. of (3.3), has no significant influence and we simply discard it. 


\section{Conclusions}

In summary, we studied the vibration properties of a suspended film bounded by a Plateau border. In free oscillations experiments, we showed that the eigenfrequency of the system varies with the cross-sectional area of the Plateau border and the geometry of the suspending films. A simplified model allowed us to predict this eigenfrequency with no adjustable parameters. Most notably, it showed that the coupling between the film and the Plateau border has to be taken into account to fully reproduce the experimental data: even in this slow oscillations, the film is not passively transported by the Plateau border.

The coupling is even more apparent in forced oscillations, and it can be seen in the amplitudes of oscillations of the film and the Plateau border, and in their phase difference. In particular, for small Plateau borders the resonant response of the system is qualitatively different than for larger Plateau borders, due to a stronger influence of the film. We reproduced this behavior by extending the model used for free oscillations.

In both situations, we could not pinpoint with certainty the main source of dissipation. In the case of free oscillations, we showed experimentally that the damping ratio does not depend on the shear viscosity of the solution. In the case of forced oscillations (which occur at larger forcing frequencies), we showed that viscous dissipation in the air is not negligible anymore, but our estimates seem to show that it remains significantly smaller than the observed damping. Other sources of dissipation are possible, including the unsteady flow in the transition regions, the flow within the Plateau border induced by its deformation, and surface dissipation. However, the study of these possible contributions is beyond the scope of our study, since they pose fundamental questions that are probably better addressed in simpler geometries. Future work will need to address this issue, of particular fundamental and practical interest.

Finally, it is worth mentioning that the model used to explain our measurements is close to the one proposed by Pierre, Dollet \& Leroy, 2014 to rationalise acoustic propagation through foams, which reinforces the idea of a key ingredient underlying the high-frequency dynamics of foams: the coupled vibration of films and Plateau borders. The main difference lies in the treatment of the surrounding air, which is enclosed in bubbles within the foam, whereas it is free to recirculate all around the films in our open system. In this respect, accounting for the role of air may be simpler in larger systems and/or higher forcing frequencies, where the soap films show several modes to which air dynamics is slaved (Kosgodagan Acharige, Elias \& Derec, 2014). Such systems, like long Plateau borders (Elias et al., 2015) are thus complementary with our setup and will probably shed further light on the vibrational response of liquid foams.

We thank Florence Elias, Caroline Derec, Cyprien Gay, Valentin Leroy and Isabelle Cantat for their insightful comments. J. P. and B. D. acknowledge support from Agence Nationale de la Recherche (ANR-11-BS09-001-Samousse), and all the members of this ANR consortium for discussions. J. S. acknowledges support from support from Région Bretagne (CREATE MOUSPORE) and Agence Nationale de la Recherche (ANR-13PDOC-0014-01-HydroSurfDyn).

\section{Appendix A. Discussion of damping}

In this appendix, we give a few details on the different sources of dissipation that we considered. As mentioned in the text, unfortunately none of them were fully compatible withe our experimental results, however we believe that they may be dominant in other configurations (for systems of larger or smaller length scales, for example). In A.1, we first 
consider the dissipation arising as films are extracted from and pulled into the Plateau border. In A.2 we then discuss the dissipation due to the movement of the air surrounding the film.

\section{A.1. Dissipation in the Plateau border region}

We discuss one possible source of dissipation of the oscillations of the Plateau border, from the viscous flow in the transition regions between the films and the Plateau border. We will recall how to compute this flow, and we will show that one consequence of this flow is a velocity-dependent modification of the surface tension of the upper and lower suspending films. We will show that this modification amounts to including a dissipative force in the equation of motion of the Plateau border, leading to a damping coefficient of the same order of magnitude as the experimental one, but not with the right scaling in viscosity.

\section{A.1.1. Velocity dependence of the traction force of a film on a Plateau border}

It is known that the traction force of a film on the Plateau border depends on their relative motion (Seiwert, Dollet \& Cantat, 2014). If a film is pulled from a reservoir (here, the Plateau border), its traction is larger than twice the equilibrium surface tension, in response to the flow generated in a transition region between the reservoir and the film. This increase depends crucially on the interfacial viscoelasticity (Denkov et al., 2005) and on the ability of surfactants to adsorb, desorb and diffuse in the bulk and along the interface (Levich, 1962). A classical assumption is the interface incompressibility, whereby the surface velocity equals the pulling velocity; this leads to the so-called Frankel's law, which determines univocally the soap film thickness as a function of the pulling velocity (Mysels, Shinoda \& Frankel, 1959), and in the frame of this hypothesis, it is easy to derive the surface tension increase.

However, such an approach is questionable in the context of our experiments, for three reasons. (i) During the oscillations, the films are alternatively withdrawn, and plunged into, the Plateau border. Contrary to the pulling situation, there is not a univocal relationship between the plunging velocity and the film thickness. However, since we are concerned with oscillations, the films that are plunging into the Plateau border were previously withdrawn from it (with the exception of the first oscillation), hence it may be assumed that at all times, the portion of the film that is successively withdrawn from, and plunging into, the Plateau border has a thickness given by Frankel's law. This hypothesis allows us to derive the surface tension decrease during plunging phases, and is at the basis of the "laid-down" film region proposed by Schwartz \& Princen, 1987 to model the effective viscosity of foams and emulsions. (ii) Frankel's law relies on a steady calculation of the transition region, which is doomed to failure at high enough frequency. (iii) The three transition regions between the Plateau border and each of the three films may not be treated as independent, and may interact through the Plateau border.

Assuming that we can apply the quasi-steady condition and neglecting the coupling between different transition regions, we remind how to derive the velocity dependence of the surface tension of a film pulled at a velocity $U$ from a Plateau border; see Cantat, 2013 for further details. It follows closely the derivation of Frankel's law (Mysels, Shinoda \& Frankel, 1959) and is also very similar to the Landau-Levich problem of plate coating (Landau \& Levich, 1942) and of the motion of a bubble in a tube (Bretherton, 1961). We focus on the transition region between the Plateau border of radius $R$ and the flat film, of thickness $2 h_{\infty}$. This region has a typical extension $\ell \approx h_{\infty} \mathrm{Ca}^{-1 / 3}$, with $\mathrm{Ca}=\eta U / \sigma$ the capillary number, which allows to use the lubrication approximation $\ell \gg h_{\infty}$ provided that $\mathrm{Ca}^{1 / 3} \ll 1$, a condition generally met in practice. The continuity of tangential stress 
at the air/liquid interface then imposes $\mathrm{d}_{x} \sigma=\left.\eta \partial_{y} v_{x}\right|_{y=h(x)}$, with $x$ the coordinate along the film and $y$ across it. The flow is driven by the gradient of Laplace pressure: $\partial_{x} p \simeq$ $\sigma \mathrm{d}_{x x x} h$, which gives: $V_{X}(X, Y)=V_{X}(X, H)+h_{\infty}^{2}\left(Y^{2}-H^{2}\right) \partial_{x} p / 2 \ell^{2}$, with $V_{X}=v_{x} / U$, $X=x / \ell, Y=y / h_{\infty}$ and $H=h / h_{\infty}$. Assuming that the interface is incompressible, we have: $V_{X}(X, H)=1$. Hence, mass conservation dictates that the flux $Q=\int_{0}^{H} V_{X} \mathrm{~d} Y=$ $\varepsilon^{3} H^{3} \mathrm{~d}_{X X X} H / \mathrm{Ca}+H$, with $\varepsilon=h_{\infty} / \ell$, is constant, and equal to 1 after the boundary conditions $V_{X} \rightarrow 1$ and $H \rightarrow 1$ as $X \rightarrow \infty$. This yields $\mathrm{d}_{x} \sigma=3 \eta U(H-1) / h_{\infty} H^{2}$. Setting $\varepsilon=(3 \mathrm{Ca})^{1 / 3}$, and assuming that the surface tension in the Plateau border retains its the equilibrium value, we get

$$
\sigma(X \rightarrow \infty)-\sigma=\sigma(3 \mathrm{Ca})^{1 / 3} \int_{-\infty}^{\infty} \frac{H-1}{H^{2}} \mathrm{~d} X,
$$

where $H$ is the solution of $\mathrm{d}_{X X X} H=(1-H) / H^{3}$ with the boundary conditions $H \rightarrow 1$, $H^{\prime}, H^{\prime \prime} \rightarrow 0$ as $X \rightarrow \infty$, which is determined numerically by a shooting method and gives: $\int_{-\infty}^{\infty}\left[(H-1) / H^{2}\right] \mathrm{d} X=1.86$. Hence, the traction of a film pulled at velocity $U$ from a reservoir is

$$
2 \sigma_{\text {pull }}=2 \sigma+3.87 \sigma \mathrm{Ca}^{2 / 3} .
$$

Let us know discuss the velocity dependence of the surface tension of the closely related case of a film plunging into the Plateau border at velocity $U$. The boundary condition on the velocity then becomes $V_{X}(X, H)=-1$. Mass conservation leads to a slightly different equation: $\mathrm{d}_{X X X} H=(H-1) / H^{3}$, and we now get: $\mathrm{d}_{x} \sigma=-3 \eta U(H-1) / h_{\infty} H^{2}$. Linearising the differential equation for $H$ around the boundary condition $H \rightarrow 1$ as $X \rightarrow \infty: H=1+\varepsilon_{H}$ gives $\mathrm{d}_{X X X} \varepsilon_{H}=\varepsilon_{H}$, which general solution compatible with the boundary condition is: $\varepsilon_{H}=\mathrm{e}^{-X / 2} \cos (X \sqrt{3} / 2+\phi)$, up to a numerical prefactor which does not modify the solution. Solving the differential equation by shooting towards decreasing values of $X$ from this linearised form gives a limit value for $\mathrm{d}_{X X} H(X \rightarrow-\infty)$ that depends on the value of $\phi$. As stated before, to guarantee that the thickness of the plunging film is the same as that of the withdrawn film, we choose $\phi$ such that this limit value equals the one obtained for the pulling case, which turns out to be: $\mathrm{d}_{X X} H(X \rightarrow-\infty)=0.643$. This fixes $\phi=1.156$, and the numerical resolution then gives: $\int_{-\infty}^{\infty}\left[(H-1) / H^{2}\right] \mathrm{d} X=0.55$. Hence, the traction of a film pushed at velocity $U$ in a reservoir is

$$
2 \sigma_{\text {push }}=2 \sigma-1.15 \sigma \mathrm{Ca}^{2 / 3}
$$

A.1.2. Damping from the dissipation in the transition regions between the films and the Plateau border

If the Plateau border is going upwards $(\dot{Z}>0)$, the magnitude of the force $f_{+}=2 \sigma_{+}$ exerted by the upper suspending film decreases, whereas that of the lower suspending film $f_{-}=2 \sigma_{-}$increases, compared to the equilibrium surface tension. The situation is reversed when the Plateau border is going downwards $(\dot{Z}<0)$. We will now show that this results in a friction force against the motion of the Plateau border. At leading order in $Z / h$, the velocity of the suspended film with respect to the Plateau border is negligible compared to those of the suspending films, hence we will consider that its surface tension does not vary. Combining (A 1), (A 2) and (3.1), we get

$$
\frac{\mu}{2 \sigma} \ddot{Z}=-\left[1+f(\dot{Z}) \mathrm{Ca}_{\dot{Z}}^{2 / 3}\right]\left(\frac{\sqrt{3}}{2}+\frac{\sqrt{3}}{4} \frac{Z}{h}\right)+\left[1+f(-\dot{Z}) \mathrm{Ca}_{\dot{Z}}^{2 / 3}\right]\left(\frac{\sqrt{3}}{2}-\frac{\sqrt{3}}{4} \frac{Z}{h}\right),
$$


where $f(\dot{Z})=1.94$ if $\dot{Z}>0$ and 0.58 if $\dot{Z}<0$, and where $\mathrm{Ca}_{\dot{Z}}=\eta|\dot{Z}| \sqrt{3} / 2 \sigma$ is the capillary number based on the relative velocity $|\dot{Z}| \sqrt{3} / 2$ (at leading order in $Z / h$ ) of the Plateau border and the suspending films. We have $f(-\dot{Z})-f(\dot{Z})=2.52 \operatorname{sign}(\dot{Z})$, hence we can rewrite the equation of motion as

$$
\frac{\mu}{2 \sigma} \ddot{Z}+\frac{\sqrt{3}}{2} 2.52 \operatorname{sign}(\dot{Z})\left(\frac{\eta|\dot{Z}| \sqrt{3}}{2 \sigma}\right)^{2 / 3}+\frac{\sqrt{3}}{2} \frac{Z}{h}=0 .
$$

This is the equation of an oscillator with a nonlinear damping, coming from the variations of surface tension due to the viscous flow in the transition region between the films and the Plateau border. With $\bar{t}=\omega_{0} t$, and $\bar{Z}=Z / h$, it becomes

$$
\partial_{\bar{t} \bar{t}} \bar{Z}+\lambda\left|\partial_{\bar{t}} \bar{Z}\right|^{2 / 3} \operatorname{sign}\left(\partial_{\bar{t}} \bar{Z}\right)+\bar{Z}=-\frac{2}{\sqrt{3}} \partial_{r} z(a, t),
$$

with a dimensionless damping coefficient

$$
\lambda=2.56 \frac{\sqrt{3}}{2}\left(\frac{\eta^{2} h}{\sigma \mu}\right)^{1 / 3}
$$

This damping coefficient is proportional to $\eta^{2 / 3}$, hence it increases by a factor 2.8 and 11 when going from a solution without glycerol to a solution containing respectively $50 \%$ and $80 \%$ of glycerol. This is not compatible with our experiments, where we have shown that the damping coefficient was barely dependent on the glycerol concentration (figure 3b), hence on the shear viscosity (table 1). Nonetheless, for the solution without glycerol, taking $\sigma=0.03 \mathrm{~N} / \mathrm{m}, h=4.8 \mathrm{~mm}$ and $s_{P}=0.3 \mathrm{~mm}^{2}$, we compute $\lambda=0.18$, which is of the same order of magnitude as the experimental values of the damping (figure $3 \mathrm{~b}$ ). As the viscosity increases, the damping coefficient estimated by (A 3) overestimates the data.

Hence, one of the two hypotheses made at the beginning of this derivation does not apply to our experiments. Either we are not in a quasi-steady situation in the transition region, but we are not aware of any prediction of the transition regions in unsteady oscillatory flow; and/or there is some coupling between the different transition regions, but this is a difficult question deserving a dedicated study, beyond the scope of this paper.

\section{A.2. Dissipation in the air}

In a thin soap film, the viscous friction in the liquid within the film is usually negligible compared to that of air (Pierre, Dollet \& Leroy, 2014, Kosgodagan Acharige, Elias \& Derec, 2014). We thus now propose an estimation of air friction on the film oscillation at the forcing frequency $f$.

Consistently with assumption $(d)$, the amplitude of oscillation of the film is small compared to its radius. In such a limit, the forces exerted by the air on the oscillating film can be deduced from the unsteady Stokes equations for the air flow (Landau \& Lifchitz, 1994). As in Sec. 3.3, it is a difficult task to solve them in our geometry, and we resort to the simple approximation consisting in identifying the film to an oscillating rigid disk, to get an estimate of air friction. At angular forcing frequency $\omega$, a frictional Stokes boundary layer entrained by the film motion develops over a thickness of order $\delta_{a}=\sqrt{2 \nu_{a} / \omega}$. In our range of forcing frequencies, this thickness is of order $0.1 \mathrm{~mm}$, which is much lower than the film radius. In this limit $\delta_{a} \ll a$, Zhang \& Stone, 1998 have proposed an asymptotic expression for the force $\mathbf{F}$ of a broadside oscillating disk; with a 
velocity $\mathbf{U} \cos \omega t$, they found

$$
\begin{aligned}
\mathbf{F} & \simeq \operatorname{Re}\left\{\mu_{a} a \mathbf{U}\left[-16\left(\frac{1}{6} \mathrm{i} \frac{\omega a^{2}}{\nu_{a}}+1.32 \mathrm{e}^{0.27 \mathrm{i} \pi} a \sqrt{\frac{\omega}{\nu_{a}}}\right)\right] \mathrm{e}^{\mathrm{i} \omega t}\right\} \\
& =\frac{8}{3} \rho_{a} a^{3} \frac{\mathrm{d}}{\mathrm{d} t}(\mathbf{U} \cos \omega t)-21.1 a^{2} \mathbf{U} \sqrt{\mu_{a} \rho_{a} \omega} \cos (\omega t+0.27 \pi) .
\end{aligned}
$$

In the first term, we recover the added mass force on the disk, already used in Sec. 3.3. The second term gives the friction force. More precisely, it contains a contribution opposite to the velocity, and an out-of-phase contribution, giving a small correction to the inertia, which we neglect. Hence, we retain a friction force: $\mathbf{F}_{a}=-21.1 \cos (0.27 \pi) \times$ $a^{2} \mathbf{U} \sqrt{\mu_{a} \rho_{a} \omega} \cos \omega t=-14.0 a^{2} \mathbf{U} \sqrt{\mu_{a} \rho_{a} \omega} \cos \omega t$.

As in Sec. 3.3, we assume that the friction is uniformly distributed on the film. Moreover, considering air friction of the portion of the film comprised between $r$ and $r+\mathrm{d} r$, we simply assume that we can replace $\mathbf{U} \cos \omega t$ by the local vertical velocity $\partial_{t} z(r, t)$. With these two approximations, the portion of film experiences a friction force of magnitude

$$
\mathrm{d} f_{a}=\frac{2 \pi r \mathrm{~d} r}{\pi a^{2}}\left[-14.0 a^{2} \sqrt{\mu_{a} \rho_{a} \omega} \partial_{t} z(r, t)\right]=-2 \times 14.0 r \sqrt{\mu_{a} \rho_{a} \omega} \partial_{t} z(r, t) .
$$

Identifying this force with the last term of the right-hand side of (5.1), we get after some algebra the following expression of $\bar{\lambda}$ in $(5.2)$

$$
\bar{\lambda}=\frac{14.0 \sqrt{\mu_{a} \rho_{a} / \omega}}{\pi \rho e+8 \rho_{a} a / 3} .
$$

In our range of forcing frequencies, $f \approx 10^{2} \mathrm{~Hz}$ and we get $\bar{\lambda} \approx 0.07$, which is five times smaller than the experimental value of the damping coefficient which fits the resonance curve, $\bar{\lambda}=0.4$. Air friction is thus not negligible, but from this estimate, it is unlikely to be the only contribution to dissipation.

\section{REFERENCES}

Afenchenko, V. O., Ezersky, A. B., Kiyashio, S. V., Rabinovich, M. I. \& Weidman, P. D. 1998 The generation of two-dimensional vortices by transverse oscillation of a soap film. Phys. Fluids 10, 390-399.

Besson, S. \& Debrégeas, G. 2007 Static and dynamics of adhesion between two soap bubbles. Eur. Phys. J. E 24, 109-117.

Boudaoud, A., Couder, Y. \& Ben Amar, M. 1999 Self-adaptation in vibrating soap films. Phys. Rev. Lett. 82, 3847-3850.

Bretherton, F. P. 1961 The motion of long bubbles in tubes. J. Fluid Mech. 10, 166-188.

Britan, A., Liverts, M. \& Ben-Dor, G. 2009 Mitigation of sound waves by wet aqueous foams. Colloids Surf. A 344, 48-55.

CAntat, I. 2013 Liquid meniscus friction on a wet plate: Bubbles, lamellae, and foams. Phys. Fluids 25, 031303.

Chaigne, A. \& Kergomard, J. 2008 Acoustique des instruments de musique. Belin.

Cohen, A., Fraysse, N., Rajchenbach, J., Argentina, M., Bouret, Y. \& Raufaste, C. 2014 Inertial mass transport and capillary hydraulic jump in a liquid foam microchannel. Phys. Rev. Lett. 112, 218303.

Cohen, A., Fraysse, N. \& Raufaste, C. 2015 High amplitude vibration of a liquid foam microchannel, in preparation.

Cohen-Addad, S., Höhler, R. \& Pitois, O. 2013 Flow in foams and flowing foams. Annu. Rev. Fluid Mech. 45, 241-267.

Couder, Y., Chomaz, J. M. \& Rabaud, M. 1989 On the hydrodynamics of soap films. Physica D 37, 384-405. 
Del Prete, E., Chinnayya, A., Domergue, L., Hadjadj A. \& HaAs, J. F. 2013 Blast wave mitigation by dry aqueous foams. Shock Waves $\mathbf{2 3}, 39-53$.

Denkov, N. D., Subramanian, V., Gurovich, D. \& Lips, A. 2005 Wall slip and viscous dissipation in sheared foams: Effect of surface mobility. Colloids Surf. A 263, 129-145.

Dollet, B. \& Raufaste, C. 2014 Rheology of aqueous foams. C. R. Physique 15, 731-747.

Drenckhan, W., Dollet, B., Hutzler, S. \& Elias, F. 2008 Soap films under large-amplitude oscillations. Philos. Mag. Lett. 88, 669-677.

Elias, F., Hutzler, S. \& Ferreira, M. S. 2007 Visualization of sound waves using regularly spaced soap films. Eur. J. Phys. 28, 755-765.

Elias, F., Janiaud, E., Bacri, J. P. \& Andreotti, B. 2014 Elasticity of a soap film junction. Phys. Fluids 26, 037101.

Elias, F., Kaurin, D., Arbogast, L., Leroy, V., Gay, C. \& Derec, C. 2015 Propagation of a transverse wave on a Plateau border, submitted to EPL.

Goldfarb, I., Orenbakh, Z., Shreiber, I. \& Vafina, F. 1997 Sound and weak shock wave propagation in gas-liquid foams. Shock Waves 7, 77-88.

Hutzler, S., SaAdatfar, M., van Der Net, A., Weaire, D. \& Cox, S. J. 2008 The dynamics of a topological change in a system of soap films. Colloids Surf. A 323, 123-131.

Joosten, J. G. H. 1984 Spectral analysis of light scattered by liquid films. I. General considerations. J. Chem. Phys. 80, 2363-2382.

Kann, K. B. 2005 Sound waves in foams. Colloids Surf. A 263, 315-319.

Kosgodagan Acharige, S., Elias, F. \& Derec, C. 2014 Vibrating soap film: origin of the dissipation. Soft Matter 10, 8341-8348.

LAmb, H. 1932 Hydrodynamics. Cambridge University Press, 6th edition.

Landau, L. \& Levich, B. 1942 Dragging of a liquid by a moving plate. Acta Physicochim. USSR 17, 42 .

Landau, L. D. \& Lifchitz, E. M. 1969 Mechanics. Pergamon Press, 2nd edition.

Landau, L. \& Lifchitz, E. 1994 Mécanique des fluides. Mir-Ellipses, 3rd edition.

Levich, V. G. 1962 Physicochemical Hydrodynamics. Prentice-Hall, Englewood Cliffs.

Moxon, N. T., Torrance, A. C. \& Richardson, S. B. 1988 The attenuation of acoustic signals by aqueous and particulate foams. Appl. Acoust. 24, 193-209.

Mujica, N. \& Fauve, S. 2002 Sound velocity and absorption in a coarsening foam. Phys. Rev. E 66, 021404

Mysels, K. J., Shinoda, K. \& Frankel, S. 1959 Soap Films: Study of Their Thinning and a Bibliography. Pergamon, New York.

Pierre, J., Dollet, B. \& Leroy, V. 2014 Resonant acoustic propagation and negative density in liquid foams. Phys. Rev. Lett. 112, 148307.

Pierre, J., Guillermic, R. M., Elias, F., Drenckhan, W. \& Leroy, V. 2013 Acoustic characterisation of liquid foams with an impedance tube. Eur. Phys. J. E 36, 113.

Rutgers, M. A., Wu, X. I., Bhagavatula, R., Petersen, A. A. \& Goldburg, W. I. 1996 Two-dimensional velocity profiles and laminar boundary layers in flowing soap films. Phys. Fluids 8, 2847-2854.

Salkin, L., Schmit, A., Panizza, P. \& Courbin, L. 2014 Influence of boundary conditions on the existence and stability of minimal surfaces of revolution made of soap films. Am. J. Phys. 82, 839-847.

Schwartz, L. W. \& Princen, H. M. 1987 A theory of extensional viscosity for flowing foams and concentrated emulsions. J. Colloid Interface Sci. 118, 201-211.

Seiwert, J., Dollet, B. \& CANTAt, I. 2014 Theoretical study of the generation of soap films: role of interfacial visco-elasticity. J. Fluid Mech. 739, 124-142.

Seiwert, J., Monloubou, M., Dollet, B. \& Cantat, I. 2013 Extension of a suspended soap film: A homogeneous dilatation followed by new film extraction. Phys. Rev. Lett. 111, 094501.

Sens, P., Marques, C. \& Joanny, J. F. 1993 Hydrodynamic modes of viscoelastic soap films. Langmuir 9, 3212-3218.

Stevenson, P. 2012 Foam Engineering: Fundamental and Applications. Wiley.

TAYLOR, G. I. 1959 The dynamics of thin sheets of fluid II. Waves on fluid sheets. Proc. R. Soc. A 253, 296-312. 
Vega, J. M., Higuera, F. J. \& Weidman, P. D. 1998 Quasi-steady vortical structures in vertically vibrating soap films. J. Fluid Mech. 372, 213-230.

Weaire, D. \& Hutzler, S. 1999 The Physics of Foams. Clarendon.

Wood, A. B. 1944 A Textbook of Sound. Bell.

Zhang, W. \& Stone, H. A. 1998 Oscillatory motions of circular disks and nearly spherical particles in viscous flows. J. Fluid Mech. 367, 329-358. 